PROCEEDINGS OF THE

AMERICAN MATHEMATICAL SOCIETY

Volume 125, Number 7, July 1997, Pages 1977-1986

S $0002-9939(97) 03723-4$

\title{
SHARP ESTIMATES FOR THE BOCHNER-RIESZ OPERATOR OF NEGATIVE ORDER IN $\mathbf{R}^{2}$
}

\author{
JONG-GUK BAK
}

(Communicated by Christopher D. Sogge)

Abstract. The Bochner-Riesz operator $T^{\alpha}$ on $\mathbf{R}^{n}$ of order $\alpha$ is defined by

$$
\left(T^{\alpha} f\right)^{\wedge}(\xi)=\frac{\left(1-|\xi|^{2}\right)_{+}^{\alpha}}{\Gamma(\alpha+1)} \hat{f}(\xi)
$$

where ${ }^{-}$denotes the Fourier transform and $r_{+}^{\alpha}=r^{\alpha}$ if $r>0$, and $r_{+}^{\alpha}=0$ if $r \leq 0$. We determine all pairs $(p, q)$ such that $T^{\alpha}$ on $\mathbf{R}^{2}$ of negative order is bounded from $L^{p}\left(\mathbf{R}^{2}\right)$ to $L^{q}\left(\mathbf{R}^{2}\right)$. To be more precise, we prove that for $0<\delta<3 / 2$ the estimate $\left\|T^{-\delta} f\right\|_{L^{q}\left(\mathbf{R}^{2}\right)} \leq C\|f\|_{L^{p}\left(\mathbf{R}^{2}\right)}$ holds if and only if $(1 / p, 1 / q) \in \Delta^{-\delta}$, where

$$
\Delta^{-\delta}=\left\{\left(\frac{1}{p}, \frac{1}{q}\right) \in[0,1] \times[0,1]: \frac{1}{p}-\frac{1}{q} \geq \frac{2 \delta}{3}, \frac{1}{p}>\frac{1}{4}+\frac{\delta}{2}, \quad \frac{1}{q}<\frac{3}{4}-\frac{\delta}{2}\right\} .
$$

We also obtain some weak-type results for $T^{\alpha}$.

The Bochner-Riesz operator $T^{\alpha}$ on $\mathbf{R}^{n}, n \geq 2$, of order $\alpha$ is a multiplier operator defined by

$$
\left(T^{\alpha} f\right)^{\wedge}(\xi)=\frac{\left(1-|\xi|^{2}\right)_{+}^{\alpha}}{\Gamma(\alpha+1)} \hat{f}(\xi)
$$

where ${ }^{\wedge}$ denotes the Fourier transform and $r_{+}^{\alpha}=r^{\alpha}$ if $r>0$, and $r_{+}^{\alpha}=0$ if $r \leq 0$. By analytic continuation this definition makes sense even when $\operatorname{Re} \alpha \leq-1$.

For our present purposes the following equivalent definition is more convenient:

$$
T^{\alpha} f=K_{\alpha} * f
$$

where $K_{\alpha}(x)=\pi^{-\alpha}|x|^{-n / 2-\alpha} J_{n / 2+\alpha}(2 \pi|x|)$ and $*$ denotes convolution. Here $J_{z}(r)$ is the Bessel function of order $z$. We refer the reader to $[\mathrm{S}]$ for the background information.

The problem of $L^{p}-L^{q}$ boundedness of $T^{-\delta}$ of negative order $-\delta$ has been studied by several authors ([Bo], [So], $[\mathrm{CS}],[\mathrm{BMO}])$. The first results in this direction were obtained by Tomas and Stein $[\mathrm{T}],[\mathrm{S}])$ : they showed that $T^{-1}$ is bounded from $L^{p}\left(\mathbf{R}^{n}\right)$ to $L^{p^{\prime}}\left(\mathbf{R}^{n}\right)$ for $p=(2 n+2) /(n+3)$ and $p^{\prime}=p /(p-1)$, which implies the sharp $L^{p}-L^{2}$ restriction theorem for the sphere $S^{n-1}$. (As is well known, $T^{-1} f$ is a multiple of $\widehat{\sigma} * f$, where $\sigma$ is the surface area measure on the sphere.)

Received by the editors October 3, 1995 and, in revised form, December 19, 1995.

1991 Mathematics Subject Classification. Primary 42B15.

The author's research was partially supported by a grant from the Pohang University of Science and Technology.

(C) 1997 American Mathematical Society 


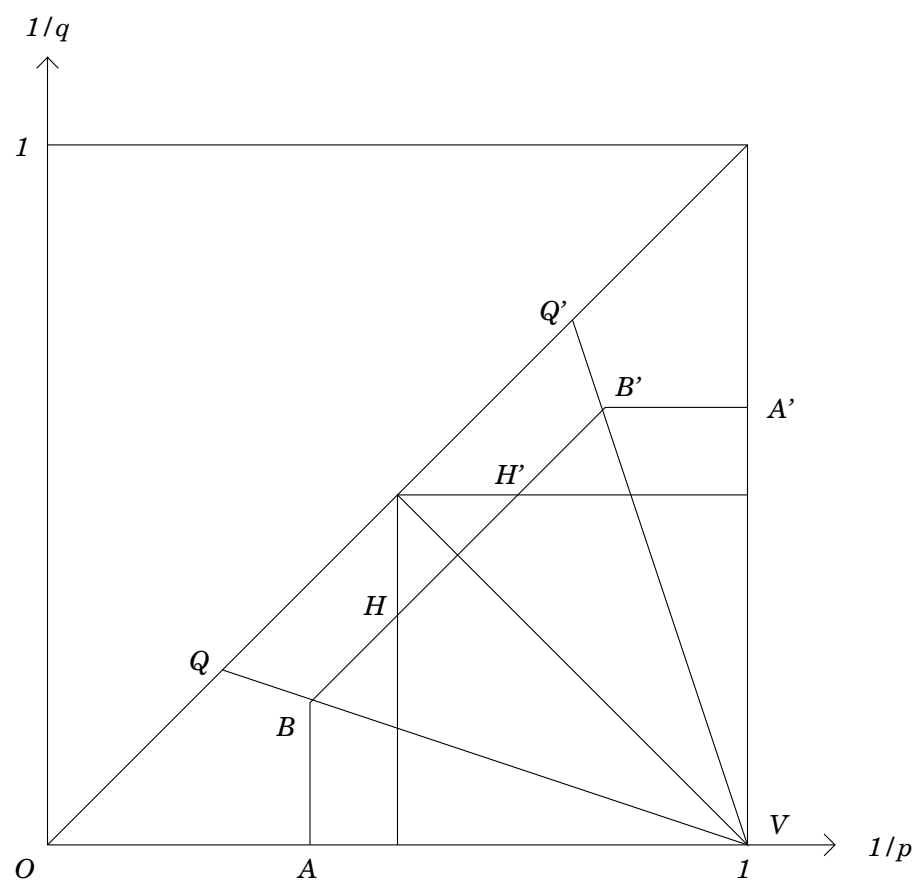

FIGURE 1

In this paper we determine all pairs $(p, q)$ such that the operator $T^{-\delta+i t}$ on $\mathbf{R}^{2}$ $(0<\delta<3 / 2)$ is bounded from $L^{p}\left(\mathbf{R}^{2}\right)$ to $L^{q}\left(\mathbf{R}^{2}\right)$. Our proof works for $t \in \mathbf{R}$, but we assume $t=0$ throughout for simplicity. (Since $K_{-3 / 2}$ is a bounded function, $T^{-3 / 2}$ is bounded from $L^{1}$ to $L^{\infty}$, but it is clearly unbounded from $L^{p}$ to $L^{q}$ for all $(p, q) \neq(1, \infty)$. When $\delta>3 / 2, T^{-\delta}$ is unbounded for any $p, q$. $)$

To describe our results we introduce some notation. (We shall take $n=2$ throughout the paper unless the dimension $n$ is explicitly mentioned.) For $0<$ $\delta<3 / 2$, define the points in the square $[0,1] \times[0,1]$ by $A=(1 / 4+\delta / 2,0), A^{\prime}=$ $(1,3 / 4-\delta / 2), B=(1 / 4+\delta / 2,1 / 4-\delta / 6), B^{\prime}=(3 / 4+\delta / 6,3 / 4-\delta / 2)$, and $V=(1,0)$. When $0<\delta<1 / 2$, also let $H=(1 / 2,1 / 2-2 \delta / 3)$ and $H^{\prime}=(1 / 2+2 \delta / 3,1 / 2)$. Note that the point $(1 / p, 1 / q)=B$ satisfies $1 / p+3 / q=1$, and so $B$ lies on the line segment $V Q$, where $Q=(1 / 4,1 / 4)$ (see Figure 1 ). Define the type set $\Delta^{-\delta}$ to be the closed solid pentagon $A B B^{\prime} A^{\prime} V$ with the closed line segments $A B$ and $A^{\prime} B^{\prime}$ removed. Namely,

$$
\Delta^{-\delta}=\left\{\left(\frac{1}{p}, \frac{1}{q}\right) \in[0,1] \times[0,1]: \frac{1}{p}-\frac{1}{q} \geq \frac{2 \delta}{3}, \frac{1}{p}>\frac{1}{4}+\frac{\delta}{2}, \frac{1}{q}<\frac{3}{4}-\frac{\delta}{2}\right\} .
$$

The analogue of this in $\mathbf{R}^{n}$ for $\alpha \neq 0$ is the set $\Delta^{\alpha}(n)$ given by

$$
\left\{\left(\frac{1}{p}, \frac{1}{q}\right): 0 \leq \frac{1}{q} \leq \frac{1}{p} \leq 1, \frac{1}{p}-\frac{1}{q} \geq \frac{-2 \alpha}{n+1}, \frac{1}{p}>\frac{n-1-2 \alpha}{2 n}, \frac{1}{q}<\frac{n+1+2 \alpha}{2 n}\right\} .
$$

It is well known ([Bo], [CS]) that for $T^{-\delta}$ to be bounded from $L^{p}\left(\mathbf{R}^{2}\right)$ to $L^{q}\left(\mathbf{R}^{2}\right)$ it is necessary that $(1 / p, 1 / q)$ is in $\Delta^{-\delta}$. The converse statement was also known to be true ([Bo], [BMO]) except possibly when $\delta<1 / 2$ and $(1 / p, 1 / q)$ was on 
either of the open line segments $B H, B^{\prime} H^{\prime}$. In particular, the problem of $L^{p_{-}}$ $L^{q}$ boundedness is completely resolved when $(1 / p, 1 / q)$ does not lie on the open segment $B B^{\prime}$. (Partial analogues of these results for $n>2$ are known; see [BMO].)

Our first result is to settle the outstanding cases by proving the boundedness of $T^{-\delta}$ whenever $(1 / p, 1 / q)$ is on the critical open segment $B B^{\prime}$. (This may be contrasted with the negative result of $\mathrm{C}$. Fefferman $[\mathrm{F}]$ at $\delta=0$.) In other words, we prove

Theorem 1. Let $0<\delta<3 / 2$. There exists a constant $C=C(p, q, \delta)$, independent of $f$, such that

$$
\left\|T^{-\delta} f\right\|_{L^{q}\left(\mathbf{R}^{2}\right)} \leq C\|f\|_{L^{p}\left(\mathbf{R}^{2}\right)}
$$

if and only if $(1 / p, 1 / q) \in \Delta^{-\delta}$.

The proof is based on an oscillatory integral estimate of Hörmander $[\mathrm{H}]$, which was originally used to give a simplified proof of the Carleson-Sjölin theorem [CaS]: if $0<\alpha \leq 1 / 2$, then $T^{\alpha}$ is bounded on $L^{p}\left(\mathbf{R}^{2}\right)$ if and only if $4 /(3+2 \alpha)<p<$ $4 /(1-2 \alpha)$.

We use a standard argument to reduce the problem to estimating $\sum_{k=1}^{\infty}\left\|T_{k}^{-\delta} f\right\|_{q}$ for characteristic functions $f=\chi_{E}$, where $T_{k}^{-\delta}$ arises from $T^{-\delta}$ by decomposing the kernel $K_{-\delta}$. The method of proof which involves an estimate like (5) below is not new (see e.g. [B, Remark 3.3]).

A similar approach also gives the following weak-type estimate on the half-open interval $A^{\prime} B^{\prime}$ which was conjectured by Carbery and Soria [CS, p. 335]:

Theorem 2. Let $0<\delta<3 / 2$. There exists a constant $C=C(p, \delta)$, independent of $f$ and $t>0$, such that

$$
\left|\left\{x \in \mathbf{R}^{2}:\left|T^{-\delta} f(x)\right|>t\right\}\right| \leq\left(\frac{C\|f\|_{p}}{t}\right)^{q}
$$

if $1 / q=3 / 4-\delta / 2$ and $3 / 4+\delta / 6<1 / p \leq 1$.

Here $|\cdot|$ denotes the Lebesgue measure. The estimate (2) is equivalent to the Lorentz space estimate

$$
\left\|T^{-\delta} f\right\|_{L^{q, \infty}\left(\mathbf{R}^{2}\right)} \leq C\|f\|_{L^{p}\left(\mathbf{R}^{2}\right)}
$$

for the same $(p, q)$ (see $[\mathrm{H}],[\mathrm{SW}])$.

Remark. (a) The question of weak type at the point $B^{\prime}$ remains open. However, a modification of the proof of Theorem 1 will yield a weaker Lorentz-Zygmund space estimate ([BR]; see also $[\mathrm{B}])$ at the point $B$ :

$$
\left\|T^{-\delta} \chi_{E}\right\|_{q} \leq C|E|^{1 / p} \log (2+|E|)
$$

if $1 / p=1 / 4+\delta / 2$ and $1 / q=1 / 4-\delta / 6$.

(b) The method of proof of Theorem 2 also yields the following weak-type results on the upper edge of the type set $\Delta^{\alpha}(n)$ :

(i) if $n=2$ and $0 \leq \alpha<1 / 2$, then $T^{\alpha}$ is of weak type $(p, q)$ when $1 / q=$ $3 / 4+\alpha / 2<1 / p \leq 1$ 
(ii) if $n \geq 3$, then $T^{\alpha}$ is of weak type $(p, q)$ for $1 / q=(n+1+2 \alpha) / 2 n$, if either

$$
\begin{gathered}
-\frac{n+1}{2}<\alpha \leq-\frac{1}{2}, \quad \frac{n+1+2 \alpha}{2 n}-\frac{2 \alpha}{n+1}<\frac{1}{p} \leq 1, \quad \text { or } \\
-\frac{1}{2}<\alpha<\frac{n-1}{2 n+2}, \quad \frac{n+3}{2 n+2} \leq \frac{1}{p} \leq 1, \quad \text { or } \\
\frac{n-1}{2 n+2} \leq \alpha<\frac{n-1}{2}, \quad \frac{n+1+2 \alpha}{2 n}<\frac{1}{p} \leq 1 .
\end{gathered}
$$

To prove (ii) we just replace the estimate (4) in the proof of Theorem 2 by the following partial analogue in $\mathbf{R}^{n}$ ([S], [So, Lemma 5.3]):

$$
\left\|G_{\lambda} f\right\|_{L^{q}\left(\mathbf{R}^{n}\right)} \leq C \lambda^{-n / q}\|f\|_{L^{r}\left(\mathbf{R}^{n}\right)}
$$

if $1 \leq r \leq 2$ and $q=(n+1) r^{\prime} /(n-1)$. (Recall that $1 / r+1 / r^{\prime}=1$.) This estimate may be derived from Theorem 1 in $[\mathrm{S}$, p. 380] by an argument similar to the one given in the appendix.

Proof of Theorem 1. By the above remarks it suffices to prove (1) when $(1 / p, 1 / q)$ lies on the open segment $B B^{\prime}$, that is, when $1 / p-1 / q=2 \delta / 3$ and $1 / 4-\delta / 6<$ $1 / q<3 / 4-\delta / 2$, for $0<\delta<3 / 2$.

Fix $0<\delta<3 / 2$. By a well known argument using an asymptotic expansion of the Bessel function, we have for some constants $c_{0}, c_{1}$

$$
T^{-\delta} f=c_{0} S_{+} f+c_{1} S_{-} f+S f
$$

where

$$
S_{ \pm} f(x)=\int_{|y| \geq 1} e^{ \pm 2 \pi i|y|}|y|^{-3 / 2+\delta} f(x-y) d y
$$

and the error term $S$ is an operator with better $L^{p}-L^{q}$ mapping properties than $S_{ \pm}$(see [S, Section IX.2]). In fact, $S$ is given by the sum of the terms $S_{ \pm}^{\prime}$, which are like $S_{ \pm}$but have lower order (i.e, with $|y|^{-5 / 2+\delta}$ in place of $|y|^{-3 / 2+\delta}$ in the integrand), and a convolution operator with a bounded $L^{1}$ kernel.

Thus, we need only to consider $S_{+}$. (Notice that $S_{-}$is the adjoint of $S_{+}$and therefore has exactly the same regularity properties.)

We now decompose $S_{+}$dyadically. Choose a function $\psi \in C_{0}^{\infty}\left(\mathbf{R}^{2}\right)$ supported in $1 / 2 \leq|y| \leq 2$ such that

$$
|y|^{-3 / 2+\delta}=\sum_{k=-\infty}^{\infty} 2^{-(3 / 2-\delta) k} \psi\left(2^{-k} y\right)
$$

when $|y|>0$. (This may be done as follows. Choose $\eta \in C_{0}^{\infty}(\mathbf{R})$ such that $\eta(t)=1$ if $0 \leq t \leq 1$, and $\eta(t)=0$ if $t \geq 2$. Then put $\psi(y)=|y|^{-3 / 2+\delta}[\eta(|y|)-\eta(2|y|)]$. This function $\psi$ has the required properties.)

Define

$$
T_{k}^{-\delta} f(x)=2^{-(3 / 2-\delta) k} \int_{|y| \geq 1} e^{2 \pi i|y|} \psi\left(2^{-k} y\right) f(x-y) d y .
$$

Then $S_{+} f=\sum_{k=0}^{\infty} T_{k}^{-\delta} f$, and so it is enough to estimate $\left\|\sum_{k=1}^{\infty} T_{k}^{-\delta} f\right\|_{q} \leq$ $\sum_{k=1}^{\infty}\left\|T_{k}^{-\delta} f\right\|_{q}$.

By an obvious change of variables we have for $k \geq 1$

$$
T_{k}^{-\delta} f(x)=2^{-(3 / 2-\delta) k} 2^{2 k} G_{2^{k}}\left(f_{2^{k}}\right)\left(2^{-k} x\right)
$$


where $f_{\lambda}(x)=f(\lambda x)$ for $\lambda>0$, and for $\lambda \geq 1$

$$
\begin{aligned}
G_{\lambda}(f)(x) & =\int_{\mathbf{R}^{2}} e^{2 \pi i \lambda|y|} \psi(y) f(x-y) d y \\
& =\int_{\mathbf{R}^{2}} e^{2 \pi i \lambda|x-y|} \psi(x-y) f(y) d y .
\end{aligned}
$$

The following is the key estimate used in our arguments:

$$
\left\|G_{\lambda} f\right\|_{L^{q}\left(\mathbf{R}^{2}\right)} \leq C \lambda^{-2 / q}\|f\|_{L^{r}\left(\mathbf{R}^{2}\right)}
$$

if $q>4$ and $3 / q+1 / r=1$ (i.e. $q=3 r^{\prime}$ ). This estimate follows from [H, Theorem 1.4]. However, for the sake of completeness we include an outline of its proof in an appendix (see also [So, Lemma 5.3]).

Since $G_{\lambda}$ is a convolution operator, by duality (4) is equivalent to the estimate

$$
\left\|G_{\lambda} f\right\|_{L^{b}\left(\mathbf{R}^{2}\right)} \leq C \lambda^{-2 / 3 b}\|f\|_{L^{a}\left(\mathbf{R}^{2}\right)}
$$

for $b>4 / 3$ and $1 / b=3(1-1 / a)$.

Now fix a point $D=(1 / p, 1 / q)$ on $B B^{\prime}$ such that $q>4$ (with $D$ close to $B$ ). Recall that we have then $1 / p=1 / q+2 \delta / 3$ and $1 / 4-\delta / 6<1 / q<3 / 4-\delta / 2$. Since $\left\|f_{\lambda}\right\|_{L^{q}\left(\mathbf{R}^{2}\right)}=\lambda^{-2 / q}\|f\|_{L^{q}\left(\mathbf{R}^{2}\right)}$, it follows from (3) and (4) that

$$
\begin{aligned}
\left\|T_{k}^{-\delta} f\right\|_{q} & =2^{-(3 / 2-\delta) k} 2^{2 k} \cdot 2^{2 k / q}\left\|G_{2^{k}}\left(f_{2^{k}}\right)\right\|_{q} \\
& \leq 2^{-(3 / 2-\delta) k} 2^{2 k} \cdot 2^{2 k / q} C 2^{-2 k / q}\left\|f_{2^{k}}\right\|_{r} \\
& =C 2^{-(3 / 2-\delta) k} 2^{2 k} 2^{-2 k / r}\|f\|_{r} \\
& =C 2^{-(3 / 2-\delta) k} 2^{6 k / q}\|f\|_{r} .
\end{aligned}
$$

Similarly, (3) and the estimate $\left(4^{\prime}\right)$, when $b=q$ and $1 / a=1-1 / 3 q$, give

$$
\begin{aligned}
\left\|T_{k}^{-\delta} f\right\|_{q} & =2^{-(3 / 2-\delta) k} 2^{2 k} \cdot 2^{2 k / q}\left\|G_{2^{k}}\left(f_{2^{k}}\right)\right\|_{q} \\
& \leq 2^{-(3 / 2-\delta) k} 2^{2 k} \cdot 2^{2 k / q} C 2^{-2 k / 3 q}\left\|f_{2^{k}}\right\|_{a} \\
& =2^{-(3 / 2-\delta) k} 2^{2 k} \cdot 2^{2 k / q} C 2^{-2 k / 3 q} 2^{-2 k / a}\|f\|_{a} \\
& =C 2^{-(3 / 2-\delta) k} 2^{2 k / q}\|f\|_{a} .
\end{aligned}
$$

From the above two estimates applied to a characteristic function $f=\chi_{E}$, we obtain

$$
\left\|T_{k}^{-\delta} \chi_{E}\right\|_{q} \leq C \min \left\{2^{-(3 / 2-\delta) k} 2^{6 k / q}|E|^{1-3 / q}, \quad 2^{-(3 / 2-\delta) k} 2^{2 k / q}|E|^{1-1 / 3 q}\right\} .
$$

Observe that the first term in the braces is smaller than the second precisely when $2^{k}<|E|^{2 / 3}$. Write $v=|E|$. For $v>0$ let $N=N(v)$ be the integer such that $2^{N}<v^{2 / 3} \leq 2^{N+1}$. Then

$$
\sum_{k=1}^{\infty}\left\|T_{k}^{-\delta} \chi_{E}\right\|_{q} \leq C \sum_{k=-\infty}^{N} 2^{k(-3 / 2+\delta+6 / q)} v^{1-3 / q}+C \sum_{k=N+1}^{\infty} 2^{k(-3 / 2+\delta+2 / q)} v^{1-1 / 3 q}
$$


Since $1 / 4-\delta / 6<1 / q<3 / 4-\delta / 2$, we have $-3 / 2+\delta+6 / q>0$, and $-3 / 2+\delta+2 / q<$ 0 . So the two geometric series are convergent, and we obtain

$$
\begin{aligned}
\sum_{k=1}^{\infty}\left\|T_{k}^{-\delta} \chi_{E}\right\|_{q} & \leq C 2^{N(-3 / 2+\delta+6 / q)} v^{1-3 / q}+C 2^{(N+1)(-3 / 2+\delta+2 / q)} v^{1-1 / 3 q} \\
& \leq C v^{(2 / 3)(-3 / 2+\delta+6 / q)} v^{1-3 / q}+C v^{(2 / 3)(-3 / 2+\delta+2 / q)} v^{1-1 / 3 q} \\
& \leq C v^{1 / q+2 \delta / 3}=C|E|^{1 / p} .
\end{aligned}
$$

Therefore, we conclude that

$$
\left\|T^{-\delta} \chi_{E}\right\|_{q} \leq C\left\|\chi_{E}\right\|_{p}
$$

if $q>4,1 / 4-\delta / 6<1 / q<3 / 4-\delta / 2$, and $1 / p=1 / q+2 \delta / 3$.

It is well known that (6) is equivalent to the Lorentz space estimate

$$
\left\|T^{-\delta} f\right\|_{q} \leq C\|f\|_{L^{p, 1}\left(\mathbf{R}^{2}\right)}
$$

for the same $(p, q)$ (see [SW, p. 195]).

Finally, by duality and interpolation in Lorentz spaces $[\mathrm{Hu}]$, [SW, p. 197]), the estimate $\left(6^{\prime}\right)$ implies (1) on the entire open segment $B B^{\prime}$. In fact, to prove (1) at any given point $\left(1 / p_{1}, 1 / q_{1}\right)$ on $B B^{\prime}$, we choose the point $D=(1 / p, 1 / q)$ on $B B^{\prime}$ sufficiently close to $B$, so that $\left(1 / p_{1}, 1 / q_{1}\right)$ lies strictly between $D$ and $D^{\prime}=\left(1 / q^{\prime}, 1 / p^{\prime}\right)$, and apply a duality and interpolation argument to the estimate $\left(6^{\prime}\right)$ at $D$.

Proof of Theorem 2. By duality (2') (or (2)) is equivalent to

$$
\left\|T^{-\delta} f\right\|_{s} \leq C\|f\|_{L^{r, 1}\left(\mathbf{R}^{2}\right)}
$$

if $1 / r=1 / 4+\delta / 2$ and $0 \leq 1 / s<1 / 4-\delta / 6$.

An immediate consequence of (4) is

$$
\left\|G_{\lambda} f\right\|_{q} \leq C \lambda^{-2 / q}\|f\|_{q}, \quad q>4 .
$$

(This follows by Hölder's inequality from the fact that if $f$ is supported near a point, then $G_{\lambda} f$ is supported near the same point.)

Fix a point $(1 / r, 1 / s)$ (on the half-open segment $A B$ ) with $1 / r=1 / 4+\delta / 2$ and $0 \leq 1 / s<1 / 4-\delta / 6$. Using (3), (8) and (4) we obtain

$$
\left\|T_{k}^{-\delta} \chi_{E}\right\|_{s} \leq C \min \left\{2^{-(3 / 2-\delta) k} 2^{2 k} 2^{-2 k / s}|E|^{1 / s}, \quad 2^{-(3 / 2-\delta) k} 2^{6 k / s}|E|^{1-3 / s}\right\} .
$$

The right-hand side is easily seen to be majorized by $C|E|^{1 / r} 2^{-\varepsilon\left|k-(1 / 2) \log _{2}\right| E||}$ if $|E|>0$, where $\varepsilon>0$ is chosen so that $1 / a+\varepsilon / 2 \leq 1 / 4+\delta / 2$ and $1 / s+\varepsilon / 6 \leq$ $1 / 4-\delta / 6$. Thus, we have

$$
\begin{aligned}
\sum_{k=1}^{\infty}\left\|T_{k}^{-\delta} \chi_{E}\right\|_{s} & \leq C|E|^{1 / r} \sum_{k=-\infty}^{\infty} 2^{-\varepsilon\left|k-(1 / 2) \log _{2}\right| E||} \\
& \leq C|E|^{1 / r} \sum_{j=-\infty}^{\infty} 2^{-\varepsilon|j|} \leq C\left\|\chi_{E}\right\|_{r}
\end{aligned}
$$

and so (7) follows. 
Appendix. (Proof of (4).) We shall prove the dual estimate $\left(4^{\prime}\right)$. Fix a function $\phi \in C_{0}^{\infty}\left(\mathbf{R}^{2}\right)$ which is supported in $|x|<1$, and put $\widetilde{\psi}(x, y)=\phi(x) \psi(x-y)$. Then $\widetilde{\psi} \in C_{0}^{\infty}\left(\mathbf{R}^{2} \times \mathbf{R}^{2}\right)$ and $\widetilde{\psi}(x, y)$ vanishes near the diagonal $x=y$. Set

$$
\widetilde{G}_{\lambda} f(x)=\int_{\mathbf{R}^{2}} e^{i \lambda|x-y|} \widetilde{\psi}(x, y) f(y) d y .
$$

The dual of the estimate (76) in [S, p. 412] (see (11) below) is a generalization of the restriction theorem for nondegenerate curves in $\mathbf{R}^{2}$ (see $\left(11^{\prime}\right)$ ), and we want to apply this "restriction theorem" to $\widetilde{G}_{\lambda} f\left(x_{1}, x_{2}\right)$ for each fixed $x_{2}$ (or $x_{1}$ ). Because of the subtlety involved in the proof, we would like to go through the individual steps closely following the exposition in [S, Chapter IX]. We cut $\widetilde{G}_{\lambda} f(x)$ into two pieces: one given by an integral over a set where the inequality $\left|x_{2}-y_{2}\right| \geq c>0$ holds, and one over a set where $\left|x_{1}-y_{1}\right| \geq c$. This can be done smoothly as follows. Choose a nonnegative function $\beta \in C^{\infty}\left(\mathbf{R}^{2} \backslash 0\right)$ which is homogeneous of degree 0 and whose values are, when $|x|=1$, given by $\beta(x)=1$ if $\left|x_{2}\right| \geq 2\left|x_{1}\right|$, and $\beta(x)=0$ if $\left|x_{2}\right| \leq\left|x_{1}\right| / 2$. For $j=1,2$ put

$$
\widetilde{G}_{j, \lambda} f(x)=\int_{\mathbf{R}^{2}} e^{i \lambda|x-y|} \widetilde{\psi}_{j}(x, y) f(y) d y
$$

where $\widetilde{\psi}_{1}(x, y)=\beta(x-y) \widetilde{\psi}(x, y)$ and $\widetilde{\psi}_{2}(x, y)=[1-\beta(x-y)] \widetilde{\psi}(x, y)$. Then $\widetilde{G}_{\lambda} f(x)=\widetilde{G}_{1, \lambda} f(x)+\widetilde{G}_{2, \lambda} f(x)$. Since $\widetilde{\psi}(x, y)$ is supported in $1 / 2 \leq|x-y| \leq 2$, we have $\left|x_{2}-y_{2}\right| \geq c$ on the support of $\widetilde{\psi}_{1}$.

The adjoint of $\widetilde{G}_{1, \lambda} f\left(\cdot, x_{2}\right)$ (with $x_{2}$ fixed) is given by

$$
T_{1, \lambda} g\left(\xi, x_{2}\right)=\int_{\mathbf{R}} e^{-i \lambda\left[\left(u_{1}-\xi_{1}\right)^{2}+\left(x_{2}-\xi_{2}\right)^{2}\right]^{1 / 2}} \widetilde{\psi}_{1}\left(u_{1}, x_{2}, \xi\right) g\left(u_{1}\right) d u_{1} .
$$

We want to get an estimate, uniform in $x_{2}$, of the $L^{q}\left(\mathbf{R}^{2}\right)$-norm of $\left(T_{1, \lambda} g\right)\left(\cdot, x_{2}\right)$. This may be done by estimating the $L^{q / 2}\left(\mathbf{R}^{2}\right)$-norm of

$$
\left(T_{1, \lambda} g\right)^{2}\left(\xi, x_{2}\right)=\int_{\mathbf{R}} \int_{\mathbf{R}} e^{-i \lambda \Phi\left(u, \xi, x_{2}\right)} \widetilde{\psi}_{1}\left(u_{1}, x_{2}, \xi\right) \widetilde{\psi}_{1}\left(u_{2}, x_{2}, \xi\right) g\left(u_{1}\right) g\left(u_{2}\right) d u_{1} d u_{2}
$$

where $\Phi\left(u, \xi, x_{2}\right)=\left[\left(u_{1}-\xi_{1}\right)^{2}+\left(x_{2}-\xi_{2}\right)^{2}\right]^{1 / 2}+\left[\left(u_{2}-\xi_{1}\right)^{2}+\left(x_{2}-\xi_{2}\right)^{2}\right]^{1 / 2}$. It turns out that in order to do this one may show that

$$
\left|\operatorname{det}\left(\frac{\partial^{2}}{\partial u_{i} \partial \xi_{j}} \Phi\left(u, \xi, x_{2}\right)\right)\right| \geq c\left|u_{1}-u_{2}\right|
$$

for some constant $c>0$ which is independent of $u, \xi$ and $x_{2}$ in the support of $\Psi\left(u, \xi, x_{2}\right)=\widetilde{\psi}_{1}\left(u_{1}, x_{2}, \xi\right) \widetilde{\psi}_{1}\left(u_{2}, x_{2}, \xi\right)$. A calculation shows that this determinant is bounded below by $c\left|x_{2}-\xi_{2}\right|^{3}\left|u_{1}-u_{2}\right|$, and hence (10) holds in the support of $\Psi$. (But (10) fails off the support of $\Psi$. This is why we had to cut $\widetilde{G}_{\lambda}$ into two pieces and consider them separately.)

To finish the estimation of the $L^{q / 2}\left(\mathbf{R}^{2}\right)$-norm of $\left(T_{1, \lambda} g\right)^{2}\left(\cdot, x_{2}\right)$, we make the change of variables $s_{1}=u_{1}+u_{2}, s_{2}=u_{1} u_{2}$. Write $\widetilde{\Phi}\left(s, \xi, x_{2}\right)=\Phi\left(u, \xi, x_{2}\right)$. Since the Jacobian $\partial\left(s_{1}, s_{2}\right) / \partial\left(u_{1}, u_{2}\right)=u_{1}-u_{2}$, we have

$$
\left(T_{1, \lambda} g\right)^{2}\left(\xi, x_{2}\right)=\int_{\mathbf{R}^{2}} e^{-i \lambda \widetilde{\Phi}\left(s, \xi, x_{2}\right)} \widetilde{\Psi}\left(s, \xi, x_{2}\right) F(s) d s
$$


where $F(s)=g\left(u_{1}\right) g\left(u_{2}\right)\left|u_{1}-u_{2}\right|^{-1}$ and $\widetilde{\Psi}\left(s, \xi, x_{2}\right)=\widetilde{\psi}_{1}\left(u_{1}, x_{2}, \xi\right) \widetilde{\psi}_{1}\left(u_{2}, x_{2}, \xi\right)$. Also, in view of the Jacobian, (10) implies that

$$
\left|\operatorname{det}\left(\frac{\partial^{2}}{\partial s_{i} \partial \xi_{j}} \widetilde{\Phi}\left(s, \xi, x_{2}\right)\right)\right| \geq c
$$

in the support of $\widetilde{\Psi}$. It follows from $\left(10^{\prime}\right)$ and $[\mathrm{H}$, Theorem 1.1] (or (79) in [S, p. 413]), which is a generalized form of the Hausdorff-Young theorem, that

$$
\left\|T_{1, \lambda} g\left(\cdot, x_{2}\right)\right\|_{L^{q}\left(\mathbf{R}^{2}\right)}^{2}=\left\|\left(T_{1, \lambda} g\right)^{2}\left(\cdot, x_{2}\right)\right\|_{L^{q / 2}\left(\mathbf{R}^{2}\right)} \leq C \lambda^{-2 /(q / 2)}\|F\|_{L^{\rho}\left(\mathbf{R}^{2}\right)}
$$

if $q>4$ and $\rho=(q / 2)^{\prime}=q /(q-2)$. It is easy to show that

$$
\|F\|_{L^{\rho}\left(\mathbf{R}^{2}\right)} \leq C\|g\|_{L^{r}(\mathbf{R})}^{2}
$$

if $q>4$ and $3 / q+1 / r=1$, by first reversing the change of variables made above, and then using Hölder's inequality and the fractional integration theorem in $\mathbf{R}^{1}$. More precisely,

$$
\begin{aligned}
\int|F(s)|^{\rho} d s & =\int\left|g\left(u_{1}\right)\right|^{\rho}\left|g\left(u_{2}\right)\right|^{\rho}\left|u_{1}-u_{2}\right|^{-\rho} d s \\
& =\int\left|g\left(u_{1}\right)\right|^{\rho} \int\left|g\left(u_{2}\right)\right|^{\rho}\left|u_{1}-u_{2}\right|^{1-\rho} d u_{2} d u_{1} \\
& \leq\left\||g|^{\rho}\right\|_{r / \rho}\left\|I_{2-\rho}\left(|g|^{\rho}\right)\right\|_{(r / \rho)^{\prime}} \\
& \leq\left\|\left.g\right|^{\rho}\right\|_{r / \rho} C\left\|\left.|| g\right|^{\rho}\right\|_{r / \rho}=C\|g\|_{r}^{2 \rho}
\end{aligned}
$$

where the last inequality follows from the fractional integration theorem: the Riesz potential $I_{a}(f)(t)=\int_{\mathbf{R}} f\left(t^{\prime}\right)\left|t-t^{\prime}\right|^{a-1} d t^{\prime}$ of order $a \in(0,1)$ is bounded from $L^{v}(\mathbf{R})$ to $L^{w}(\mathbf{R})$ if $1 / v-1 / w=a$ and $1<v<w<\infty$. Since $\rho=(q / 2)^{\prime}$, the equation $1 /(r / \rho)-1 /(r / \rho)^{\prime}=2-\rho$ can be simplified to $3 / q+1 / r=1$.

Thus we get

$$
\left\|T_{1, \lambda} g\left(\cdot, x_{2}\right)\right\|_{L^{q}\left(\mathbf{R}^{2}\right)} \leq C \lambda^{-2 / q}\|g\|_{L^{r}(\mathbf{R})}
$$

if $q>4$ and $3 / q+1 / r=1$, where the constant $C$ is independent of $x_{2}$ and of course $\lambda$ and $g$. (This is a variant of the estimate (76) in [S, p. 412]) mentioned above.)

Dualizing (11) gives

$$
\left(\int_{\mathbf{R}}\left|\widetilde{G}_{1, \lambda} f\left(x_{1}, x_{2}\right)\right|^{b} d x_{1}\right)^{1 / b} \leq C \lambda^{-2 / 3 b}\|f\|_{L^{a}\left(\mathbf{R}^{2}\right)}
$$

if $b>4 / 3$ and $1 / b=3(1-1 / a)$. (This is a "generalized restriction theorem".) Raising both sides of $\left(11^{\prime}\right)$ to the $b$-th power and integrating in $x_{2}$ (over a compact set, since $\widetilde{\psi}$ is compactly supported), we obtain

$$
\int_{\mathbf{R}^{2}}\left|\widetilde{G}_{1, \lambda} f(x)\right|^{b} d x \leq C \lambda^{-2 / 3}\|f\|_{L^{a}\left(\mathbf{R}^{2}\right)}^{b} .
$$

To get an estimate for $\widetilde{G}_{2, \lambda} f(x)$, we just reverse the roles of $x_{1}$ and $x_{2}$, that is, we fix $x_{1}$ instead of $x_{2}$ and repeat the above argument. Adding the estimates for $\widetilde{G}_{1, \lambda}$ and $\widetilde{G}_{2, \lambda}$, we get

$$
\int_{\mathbf{R}^{2}}\left|\widetilde{G}_{\lambda} f(x)\right|^{b} d x \leq C \lambda^{-2 / 3}\|f\|_{L^{a}\left(\mathbf{R}^{2}\right)}^{b} .
$$


Note that, by translation invariance of Lebesgue measure, the last estimate remains unchanged if $\widetilde{\psi}(x, y)$ in (9) is replaced by $\widetilde{\psi}\left(x-x^{0}, y-x^{0}\right)$ for $x^{0} \in \mathbf{R}^{2}$. To see this, just observe that

$$
\int_{\mathbf{R}^{2}} e^{i \lambda|x-y|} \widetilde{\psi}\left(x-x^{0}, y-x^{0}\right) f(y) d y=\widetilde{G}_{\lambda}\left(f_{x^{0}}\right)\left(x-x^{0}\right)
$$

where $f_{x^{0}}(y)=f\left(y+x^{0}\right)$.

This implies that, since $\phi(x)$ is supported in $|x|<1$, and $\psi(y)$ in $|y| \leq 2$,

$$
\int\left|\phi\left(x-x^{0}\right) G_{\lambda} f(x)\right|^{b} d x \leq C \lambda^{-2 / 3}\left(\int_{\left|y-x^{0}\right|<3}|f(y)|^{a} d y\right)^{b / a} .
$$

We integrate both sides in $x^{0}$ and use Minkowski's inequality, noting that $b / a \geq 1$, to obtain

$$
\begin{aligned}
& \|\phi\|_{b}^{b} \int_{\mathbf{R}^{2}}\left|G_{\lambda} f(x)\right|^{b} d x \leq C \lambda^{-2 / 3}\left\|\int \chi_{B\left(x^{0}, 3\right)}(y)|f(y)|^{a} d y\right\|_{L^{b / a}\left(d x^{0}\right)}^{b / a} \\
& \quad \leq C \lambda^{-2 / 3}\left(\int\left\|\chi_{B(y, 3)}\left(x^{0}\right)|f(y)|^{a}\right\|_{L^{b / a}\left(d x^{0}\right)} d y\right)^{b / a} \leq C \lambda^{-2 / 3}\|f\|_{a}^{b}
\end{aligned}
$$

where $\chi_{B\left(x^{0}, 3\right)}(y)$ is the characteristic function of the disc of center $x^{0}$ and radius 3 . This completes the proof of $\left(4^{\prime}\right)$ and $(4)$.

We thank the referee for many valuable comments and suggestions. These greatly improved the presentation of our results. He also informed us that A. Seeger [Se] has recently proved that $T^{\alpha}$ on $\mathbf{R}^{2}, 0<\alpha \leq 1 / 2$, is of weak type $(p, p)$ at the critical exponent $p=4 /(3+2 \alpha)$. (See [Se] for more references.)

\section{REFERENCES}

[B] J.-G. Bak, Sharp convolution estimates for measures on flat surfaces, J. Math. Anal. Appl. 193 (1995), 756-771. CMP 95:15

[BMO] J.-G. Bak, D. McMichael, and D. Oberlin, $L^{p}-L^{q}$ estimates off the line of duality, J. Austral. Math. Soc. (Series A) 58 (1995), 154-166. MR 96j:42004

[BR] C. Bennett and K. Rudnick, On Lorentz-Zygmund spaces, Dissertationes Math. 175 (1980), 1-67.

[Bo] L. Börjeson, Estimates for the Bochner-Riesz operator with negative index, Indiana U. Math. J. 35 (1986), 225-233. MR 87f:42036

[CS] A. Carbery and F. Soria, Almost-everywhere convergence of Fourier integrals for functions in Sobolev spaces, and an $L^{2}$-localisation principle, Rev. Mat. Iberoamericana 4 (1988), 319-337. MR 91d:42015

[CaS] L. Carleson and P. Sjölin, Oscillatory integrals and a multiplier problem for the disc, Studia Math. 44 (1972), 287-299. MR 50:14052

[F] C. Fefferman, The multiplier problem for the ball, Ann. of Math. 94 (1971), 330-336. MR 45:5661

[H] L. Hörmander, Oscillatory integrals and multipliers on $F L^{p}$, Ark. f. Mat. 11 (1973), 1-11. MR 49:5674

[Hu] R. Hunt, On $L(p, q)$ spaces, L'Ens. Math. 12 (1966), 249-275. MR 36:6921

[Se] A. Seeger, Endpoint inequalities for Bochner-Riesz multipliers in the plane, Pacific J. Math. 174 (1996), 543-553.

[So] C. D. Sogge, Oscillatory integrals and spherical harmonics, Duke Math. J. 53 (1986), 43-65. MR 87g:42026

[S] E. M. Stein, Harmonic analysis: Real-variable methods, orthogonality, and oscillatory integrals, Princeton Univ. Press, Princeton, New Jersey, 1993. MR 95c:42002 
[SW] E. M. Stein and G. Weiss, An introduction to Fourier analysis on Euclidean spaces, Princeton Univ. Press, Princeton, New Jersey, 1971.

[T] P. Tomas, A restriction theorem for the Fourier transform, Bull. Amer. Math. Soc. 81 (1975), 477-478. MR 50:10681

Department of Mathematics, Florida State University, Tallahassee, Florida 32306 3027

Current address: Department of Mathematics, Pohang University of Science and Technology, Pohang 790-784, Korea

E-mail address: bak@euclid.postech.ac.kr 